\title{
Inhibition of BAMBI reduces the viability and motility of colon cancer via activating TGF- $/$ /Smad pathway in vitro and in vivo
}

\author{
WENZHEN YU and HONG CHAI
}

Department of Gastroenterology, The People's Hospital of Wuhan University, Wuchang, Wuhan, Hubei 430060, P.R. China

Received March 6, 2017; Accepted August 3, 2017

DOI: $10.3892 / \mathrm{ol} .2017 .6811$

\begin{abstract}
Colon cancer is a highly metastatic gastrointestinal cancer. BMP activin membrane-bound inhibitor (BAMBI), as a pseudo-receptor of the tumor growth factor (TGF)- $\beta$ signal transduction pathway, has previously been demonstrated to be involved in human cancers. The present study demonstrated that BAMBI-small interfering (si)RNA regulated the viability and motility of colon cancer by activating TGF- $\beta$ signaling. The expression level of BAMBI was suppressed by transfecting BAMBI-siRNA into the SW480 and HT-29 colon cancer cell lines. Decreased cell proliferation and increased cell apoptosis were detected in SW480 and HT-29 cells transfected with BAMBI-siRNA. Decreased expression of proliferation marker proteins Ki67 and proliferating cell nuclear antigen and elevated expression of apoptosis marker proteins (caspases-3, -8 and -9 ) further verified the role of BAMBI-siRNA in inhibiting cell viability. Silencing of BAMBI strongly reduced the closing rate and the number of invasive cells compared with control group. BAMBI-siRNA additionally resulted in decreased expression of migration marker proteins matrix metalloproteinase-9 (MMP-9), MMP-14 and vascular endothelial cell growth factor. In addition, the expression of TGF- $\beta$ and phosphorylated-mothers against decapentaplegic homolog (Smad) 2/3 was increased in W480 and HT-29 cells transfected with BAMBI-siRNA. Elevated expression of the downstream signaling molecule E2F transcription factor 4/5 and suppressed c-MYC were additionally detected in the BAMBI-siRNA group. Finally, the in vivo experiment in the CSC xenograft revealed that BAMBI-siRNA strongly reduced
\end{abstract}

Correspondence to: Professor Wenzhen Yu, Department of Gastroenterology, The People's Hospital of Wuhan University, 238 Jiefang Road, Wuchang, Wuhan, Hubei 430060, P.R. China E-mail: yuwenzhenwhu@163.com

Abbreviations: BAMBI, BMP activin membrane-bound inhibitor; TGF- $\beta$, transforming growth factor- $\beta$; PCNA, proliferating cell nuclear antigen; siRNA, small interfering RNA; CRC, colorectal cancer; MMP, matrix metalloproteinase; VEGF, vascular endothelial cell growth factor; TGFBR2, type II TGF- $\beta$ receptor

Key words: BAMBI, colon cancer, viability, motility, TGF- $\beta /$ Smad pathway the tumor growth and tumor volume. BAMBI-siRNA inhibited hepatic metastases and the expression of metastasis-associated proteins. The upregulated expression of TGF- $\beta$ signaling proteins were detected in the BAMBI-siRNA group compared with control group in vivo. Overall, the results of the present study indicated that the inhibition of BAMBI reduces the viability and motility of colon cancer and may involve activation of the TGF- $\beta /$ Smad pathway in vitro and in vivo.

\section{Introduction}

Colon cancer (as named as colorectal cancer, CRC) is the third most prevalent gastrointestinal cancer in the world, marked by liver metastasis and high recurrence rate $(1,2)$. Treatment of CRC is largely ineffective due to lack of thorough understanding of its pathogenesis, resulting in high morbidity and mortality $(3,4)$. Therefore, getting a better understanding of the underlying molecular pathogenesis of CRC will help to develop more effective therapies for the treatment of CRC.

BMP activin membrane-bound inhibitor (BAMBI) is a trans-membrane glycoprotein located at chromosomal 10p11.2-p12.3. BAMBI is also named as pseudo-receptor due to its similar structure with extracellular domain of type I receptors of the transforming growth factor- $\beta$ (TGF- $\beta$ ) family, activin, and bone morphogenetic protein (BMP) (5). Previous studies suggested that BAMBI may be involved in a variety of diseases, especially in cancer. BAMBI was firstly found down-regulated in human melanoma (6) and then was identified to induce cell growth and invasion in human gastric carcinoma via negatively regulating TGF- $\beta$ signaling (7). Moreover, aberrantly elevated level of BAMBI was found in most colorectal and hepatocellular carcinomas (8). As a pseudo-receptor of TGF- $\beta$ signal transduction pathway, the negative feedback regulation of BAMBI in the signal transduction pathway of TGF- $\beta$ has been widely confirmed. However, the exact molecular mechanism of the interaction between BAMBI and TGF- $\beta /$ Smad pathway in the viability and motility of colon cancer has yet been poorly defined.

According to previous reports, the TGF- $\beta /$ Smad signaling pathway is one of the most commonly related pathways in human cancers. The TGF- $\beta /$ Smad signaling pathway inhibits or promotes the development of tumors in different stages. For example, TGF- $\beta$ gene inhibits the development of breast cancer at early stage by inhibiting cell-cycle progression and tumor growth. However, TGF- $\beta$ signaling is propitious to 
carcinoma cell invasiveness and metastasis during late-stage tumorigenesis $(9,10)$. It has been reported that TGF- $\beta$ signaling suppresses the growth of multiple epithelial cell types, but acts as a tumor promoter in colorectal cancer due to the mutational inactivation of type II TGF- $\beta$ receptor (TGFBR2), SMAD2, and SMAD4 $(11,12)$. Till now, the complex regulatory mechanism of TGF- $\beta$ signaling in the development of CRC has not been clearly elucidated till now.

In this study, we aimed to explore the mechanism of BAMBI in the pathogenesis of colorectal cancer. BAMBI siRNA was verified to restrain cell proliferation and promote cell apoptosis in vitro. BAMBI siRNA also suppressed the migration and invasion of CRC. The in vivo experiment in CSC xenograft further convinced the inhibitory effect of BAMBI siRNA in tumor growth and metastasis that may activate the TGF- $\beta /$ Smad signaling. Our study revealed the regulatory mechanism of BAMBI in pathogenesis of colorectal cancer and provided new targets for cancer therapy.

\section{Materials and methods}

Cell lines culture. Human colon cancer cell lines SW480 and HT-29 were purchased from American Type Culture Collection (Manassas, VA, USA). Both of the cell lines were maintained routinely in RPMI-1640 (cat no. 11875-093; Gibco, Grand Island, NY, USA) supplemented with $10 \%$ fetal bovine serum (Life Technologies, Inc., Grand Island, NY, USA) and grown at $37^{\circ} \mathrm{C}$ in a $5 \% \mathrm{CO}_{2}$ cell culture incubator.

Cell transfection. SiRNA fragments targeting BAMBI were designed and purchased from Invitrogen (Carlsbad, CA, USA). The control and scramble fragments were designed as the negative control of BAMBI. The SW480 and HT-29 cells were seeded in 24-well plates at $1 \times 10^{5}$ cells per well. BAMBI-siRNA and siRNA-scramble and control were transfected into SW480 and HT-29 cells using Lipofectamine 2000 according to the protocol (Invitrogen). Cells were harvested for subsequent experiments after transfection for 24

Western blotting. Cell samples were lysed in lysis buffer (Beyotime, Jiangsu, China). $30 \mu \mathrm{g}$ protein was separated through SDS-PAGE and then was transferred onto Polyvinylidene Fluoride membranes (Millipore, Billerica, MA, USA). The membranes were then blocked in PBST (PBS with $0.1 \%$ Tween 20 ) containing 5\% nonfat milk for $2 \mathrm{~h}$ at room temperature, and then were incubated with the primary antibodies: Anti-BAMBI, anti-Ki67, antiproliferating cell nuclear antigen (PCNA), anti-caspase-3, anti-caspase-8, anti-caspase-9, anti- matrix metalloproteinase (MMP)-9, anti-MMP-14, anti-VEGF, anti-TGF- $\beta$, anti-Smad2/3, anti-p-Smad2/3, anti-E2F4/5, anti-c-MYC, anti-GAPDH (Abcam, Cambridge, UK) and the corresponding HRP-conjugated secondary antibodies. Proteins were detected using a ChemiDoc XRS imaging system and Quantity One analysis software (Bio-Rad, San Francisco, CA, USA). GAPDH (Abcam) was used as endogenous reference.

Cell proliferation assay. Cell proliferation was assayed using the cell counting Kit-8 (CCK-8; Dojindo Laboratories, Tokyo, Japan) according to the manufacturer's protocol. A total of approximately $5 \times 10^{3}$ cells were seeded onto 96 -well plates, followed by pretreated with BAMBI-siRNA or siRNA-scramble or control, respectively. Cells were then incubated with CCK-8 solution for $2 \mathrm{~h}$ at $37^{\circ} \mathrm{C}$. The absorbance was measured at $450 \mathrm{~nm}$ using multifunctional microplate reader spectraMaxM5 (Molecular Devises, Sunnyvale, CA, USA) at indicated time points. All experiments were repeated at least three times.

Flow cytometric analysis of cell apoptosis. Cells in each group were harvested at $48 \mathrm{~h}$ post-transfection. For the apoptosis analysis, cells were re-suspended $\left(1 \times 10^{6}\right)$ and fixed, then were stained using the Annexin V-fluorescein isothiocyanate (FITC) and PI apoptosis detection kits (Annexin V-FITC Apoptosis Detection kit; Thermo Fisher Scientific, Waltham, MA, USA). Then the apoptosis rates were analyzed using the FACS Caliber II sorter and Cell Quest FACS system (BD Bio-sciences, San Jose, CA, USA) according to the manufacturer's protocols. The flow cytometry analysis was repeated at least three times.

Wound-healing assay. Wound-healing assay was performed to evaluate the migration rate of SW480 and HT-29 cells transfected with BAMBI-siRNA or siRNA-scramble or control. Firstly, $1.5 \times 10^{6}$ cells/well were seeded in 6-well plates and cultured overnight until the cells reached $90 \%$ confluence. A straight scratch was created by a sterile pipette tip. The destroyed cells were rinsed off with PBS 3 times gently and cultured in medium for another $24 \mathrm{~h}$. Cell migration was observed and imaged at 0 and $24 \mathrm{~h}$ with a digital camera (Leica DFC300FX).

Transwell invasion assay. For the invasion assays, SW480 and HT-29 cells pre-transfected with BAMBI siRNA or siRNA scramble or control $\left(2 \times 10^{4}\right.$ cells/well) were placed in transwell cell culture chambers ( $8 \mathrm{~mm}$ pore size; Merck Millipore Corp, Billerica, MA,USA), coating with Matrigel (Becton-Dickinson, East Rutherford, NJ, USA). Cell suspension was placed in the upper chamber of the insert and lower chamber was filled with complete medium (containing 20\% FBS) as a chemoattractant. Cells were incubated for another $24 \mathrm{~h}$. Non-invading cells on the upper membranes were removed and the invasive cells were fixed in $95 \%$ ethanol, stained with hematoxylin. Cells were examined, photographed and quantified under a light microscope at 100x in five random fields per membrane. Each sample was assayed in triplicate.

Subcutaneously xenografted mouse model. All animal experiments were carried out in accordance with a protocol approved by the Institutional Animal Care and Use Committee (IACUC). HT-29 cells were transfected with BAMBI siRNA or siRNA scramble or control for $24 \mathrm{~h}$. Then, $4 \times 10^{6}$ cells were subcutaneously inoculated into 6-8 weeks old male athymic nude mice. After tumors (100-150 $\left.\mathrm{mm}^{3}\right)$ had established, the tumor volume was measured every 5 days with a caliper, and calculated in length $\mathrm{x}\left(\mathrm{width}^{2}\right) / 2$.

Ex vivo fluorescence imaging of the liver. Fluorescence in livers from colon cancer xenografts mice was observed using the Xenogen IVIS spectrum imager (Caliper Life Sciences, Inc., 
A

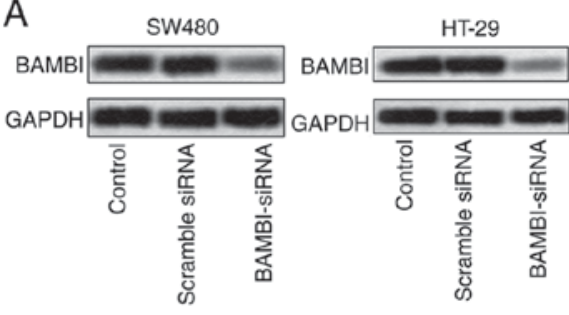

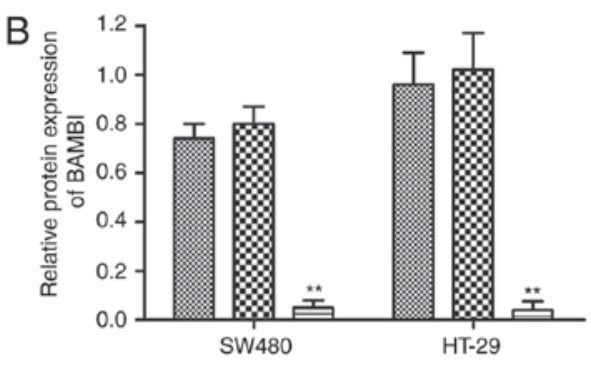

E

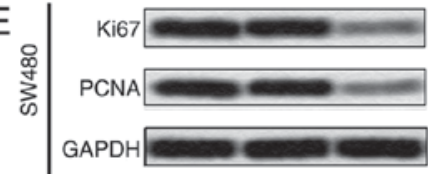

C sw 480

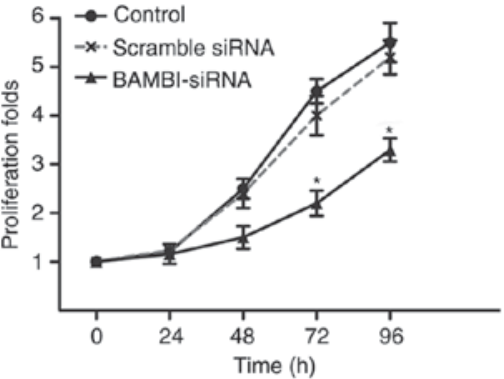

D ${ }^{\text {HT }}-29$
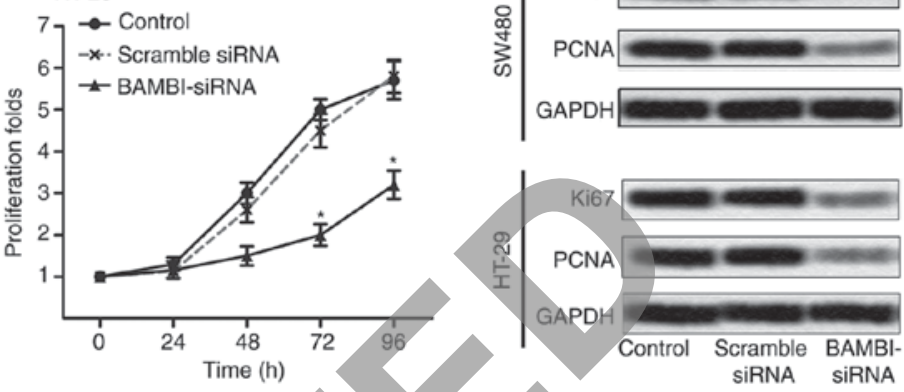

F SW480

$-2$

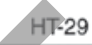

m. Scramble siRNA

曰BAMBI-siRNA
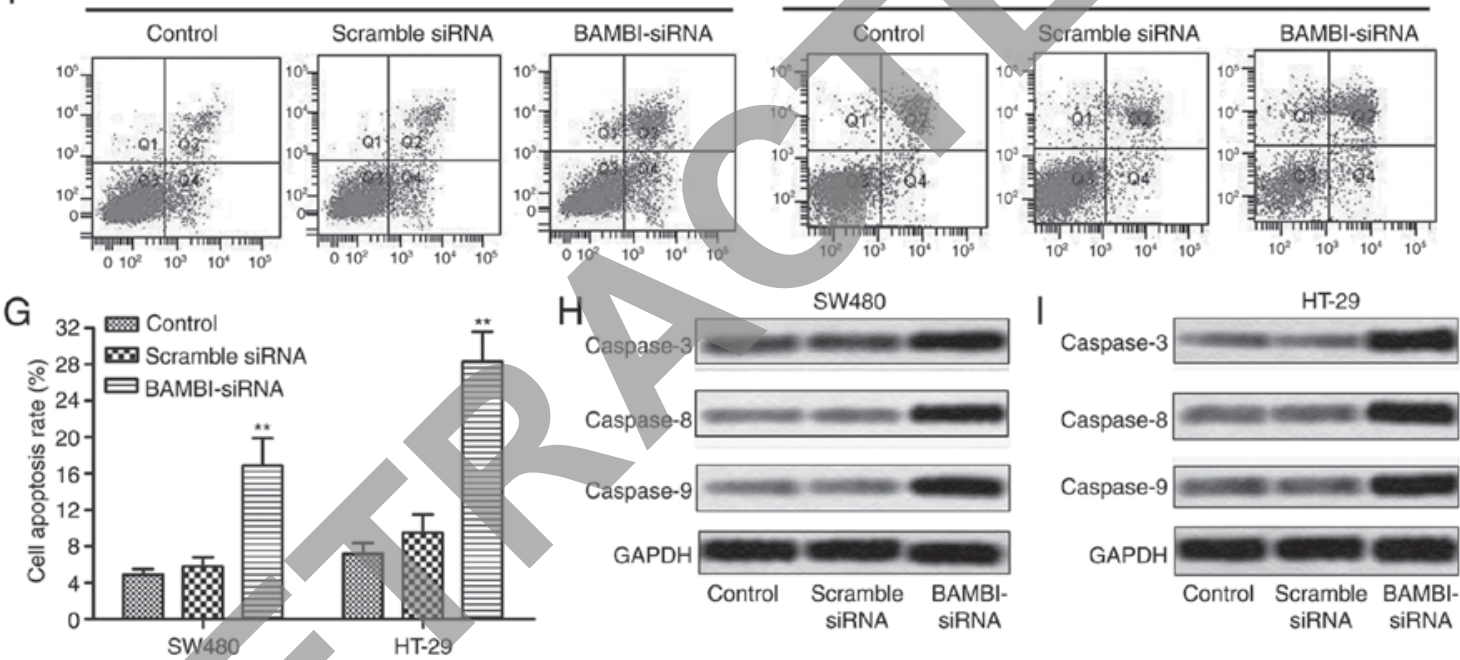

Figure 1. The inhibition of BAMBI reduces cell viability of colorectal cancer. CRC cell lines SW480 and HT-29 were transfected with BAMBI-siRNA or siRNA-scramble or control, respectively. (A) The expression of BAMBI in SW480 and HT-29 cells was detected through western blotting. GAPDH was used as an endogenous reference. (B) Histogram represents the statistical analysis of the changes in the expression of proteins $\left({ }^{* *} \mathrm{P}<0.01\right.$ vs. control group). (C and D) CCK8 assay was conducted to detected cell proliferation in SW480 and HT-29 cells ("P $<0.05$ vs. control group). (E) The expression of proliferation marker proteins (Ki67 and PCNA) in SW480 and HT-29 cells was valued through western blotting. GAPDH was used as an endogenous reference. (F) Cell apoptosis rate in SW480 and HT-29 cells was detected through flow cytometric analysis. (G) Histogram represents the statistical analysis of flow cytometric (" $\mathrm{P}<0.01$ vs. control group). (H and I) The expression of apoptosis marker proteins (caspase-3, caspase-8, caspase-9) in SW480 and HT-29 cells was detected through western blotting. GAPDH was used as an endogenous reference. The bars showed means \pm SD of three independent experiments.

Hopkinton, MA, USA). The total signal intensity was quantified by drawing the region of interest (ROI) using the matching analysis software package supplied by the manufacturer.

Immunohistochemistry. Formalin-fixed paraffin-embedded sections $(5 \mu \mathrm{M})$ from tissue microarrays were prepared. They were deparaffinized in xylene and rehydrated then were incubated in $30 \% \mathrm{H}_{2} \mathrm{O}_{2}$ to quench the activity of endogenous peroxidase. Then the sections were incubated with primary antibodies directed against Ki67 and MMP-14 overnight at $4^{\circ} \mathrm{C}$. Proteins were visualized under a light microscopy.

Statistical analysis. All results are presented as mean $\pm \mathrm{SD}$ and evaluated with a Student's t-test. $\mathrm{P}<0.05$ was considered to indicate a statistically significant difference. All experiments were performed at least three times performed in triplicate.

\section{Results}

The inhibition of BAMBI reduces cell viability of colorectal cancer. To explore the effect of BAMBI on cell viability of CRC, SW480 and HT-29 cells were transfected with BAMBI-siRNA or siRNA-scramble or control, respectively. As shown in Fig. 1A and $\mathrm{B}$, the expression of BAMBI was largely down-regulated in BAMBI-siRNA group compared with control group in SW480 and HT-29 cells ( $\left.{ }^{* *} \mathrm{P}<0.01\right)$. Then, CCK8 assay was conducted to detected cell proliferation in SW480 and HT-29 cells. In the first $24 \mathrm{~h}$, no obvious difference was detected among the groups, 
A
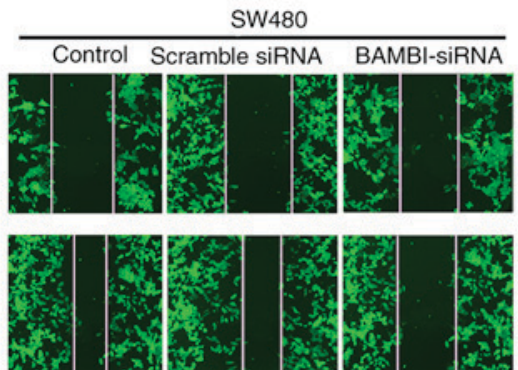

C

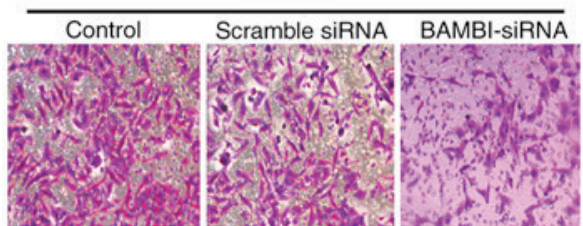

E

SW480

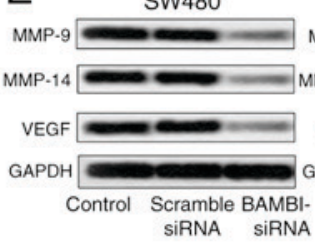

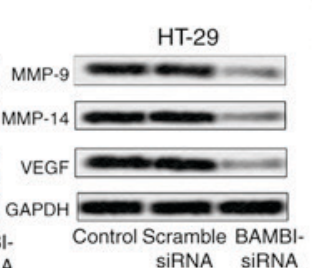

SIRNA SIRNA
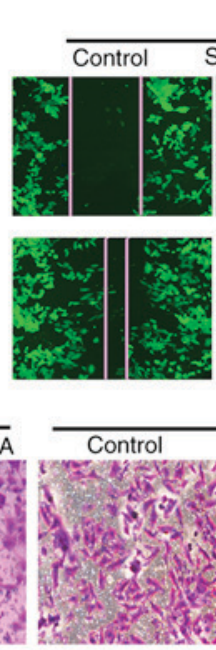

HT-29
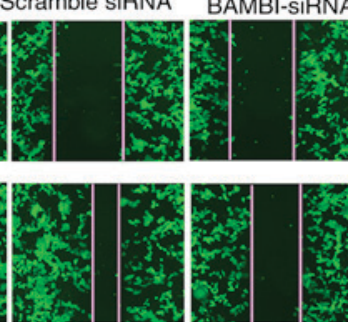

HT-29

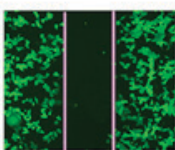

Scramble siRN
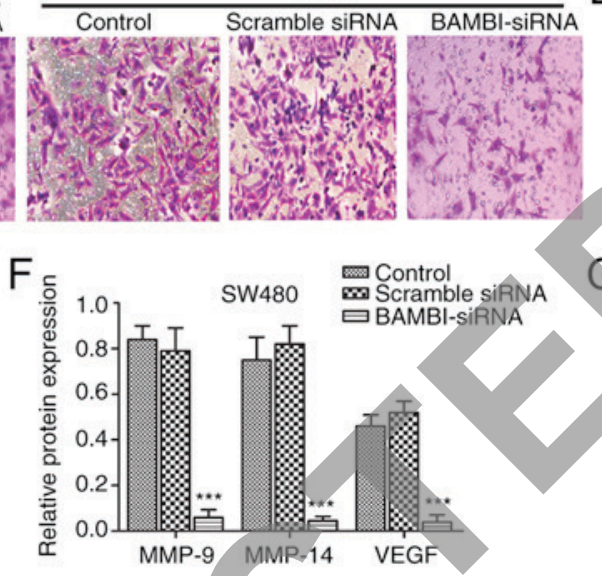

B

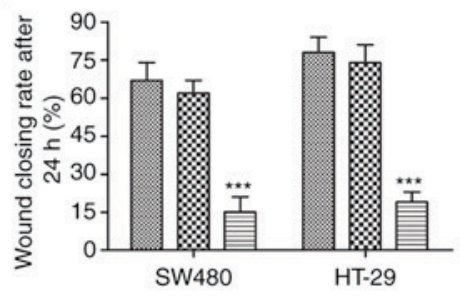

$\mathrm{D}$

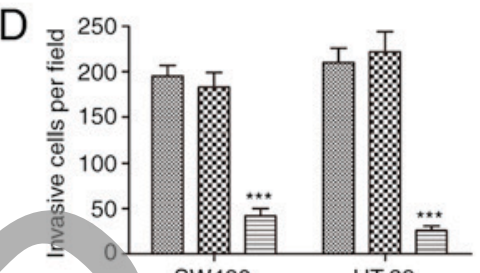

SW480

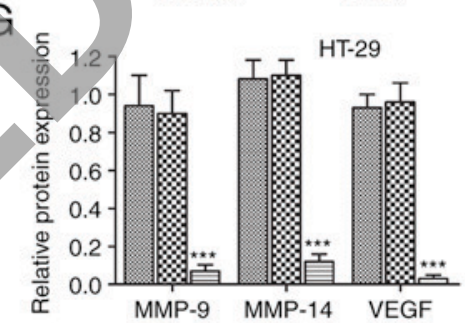

Figure 2. The inhibition of BAMBI reduces cell motility of colorectal cancer. CRC cell lines SW480 and HT-29 were transfected with BAMBI-siRNA or siRNA-scramble or control, respectively. (A) The migration rate of SW480 and HT-29 cells pre-treated as described for 24 h was observed through wound-healing assays. (B) Histogram represents the statistical analysis of wound-healing assays $\left({ }^{* * *} \mathrm{P}<0.001\right.$ vs. control group). (C) Trans-well invasion assays were conducted to observe the invasive cells in SW480 and HT-29 cells. (D) Histogram represents the statistical analysis of trans-well invasion assays ( ${ }^{* * *} \mathrm{P}<0.001$ vs. control group). (E) The expression of tumor metastasis-related proteins (MMP-9/MMP-14/VEGF) in SW480 and HT-29 cells was detected through western blotting. GAPDH was used as an endogenous reference. (F and G) Histogram represents the statistical analysis of the changes in the expression of metastasis-related proteins. The bars showed means \pm SD of three independent experiments.

but a growing gap was shown in subsequent $24-96 \mathrm{~h}$ between the BAMBI-siRNA group and control groups $\left({ }^{*} \mathrm{P}<0.05\right.$, Fig. $1 \mathrm{C}$ and D). The suppressed level of proliferation marker proteins (Ki67 and PCNA) by BAMBI-siRNA transfection further convinced that BAMBI-siRNA inhibited proliferation of CRC cells (Fig. 1E). Moreover, flow cytometric analysis indicated that cell apoptosis rate was promoted in BAMBI-siRNA group compared with control group in SW480 and HT-29 cells $\left({ }^{* *} \mathrm{P}<0.01\right.$, Fig. $1 \mathrm{~F}$ and $\left.\mathrm{G}\right)$. The expression of apoptosis marker proteins (caspase-3, caspase-8, caspase-9) was also increased by BAMBI-siRNA transfection compared with control group (Fig. 1H and I). These results indicated that the inhibition of BAMBI reduced cell viability of CRC.

The inhibition of BAMBI reduces cell motility of colorectal cancer. Having known that suppressed BAMBI reduced cell viability of CRC, a serious of experiments were then conducted to explore the effect of BAMBI on cell motility of CRC. The results of wound healing assays showed a large closure of the gap in the control group and siRNA-scramble group, whereas, a negligible effect on the closing rate of scratch wounds was seen in the BAMBI-siRNA group compared with control group $\left({ }^{* * *} \mathrm{P}<0.001\right.$, Fig. $2 \mathrm{~A}$ and B). Besides that, the number of invasive cells was decreased over 4 times in SW480 and HT-29 cells transfected with BAMBI-siRNA compared with control group $\left({ }^{* * *} \mathrm{P}<0.001\right.$, Fig. $2 \mathrm{C}$ and $\left.\mathrm{D}\right)$. Moreover, the expression level of tumor metastasis-related proteins (MMP-9, MMP-14 and VEGF) were largely decreased by BAMBI-siRNA $\left({ }^{* * *} \mathrm{P}<0.001\right.$, Fig. 2E-G). These results indicated that the inhibition of BAMBI reduced cell motility of colorectal cancer.

The inhibition of BAMBI activates TGF- $\beta /$ Smad signaling. Considering the universal correlation of TGF- $\beta / \mathrm{Smad}$ signaling and BAMBI, the interaction between the two in CRC was further explored. The expression of TGF- $\beta$ was obviously up-regulated in SW-480 cells transfected with BAMBI-siRNA. No significant change was observed in the expression of substrate $\operatorname{Smad} 2 / 3$ but the level of $\mathrm{p}-\mathrm{Smad} 2 / 3$ was largely increased by BAMBI-siRNA. Elevated expression of downstream signaling molecule E2F4/5 and suppressed c-MYC were also detected in the BAMBI-siRNA group. The same changes in protein expression were also showed in HT-29 cells ( ${ }^{* *} \mathrm{P}<0.01$, Fig. $\left.3 \mathrm{~A}-\mathrm{F}\right)$. The results above indicated that the inhibition of BAMBI activated TGF- $\beta /$ Smad signaling in CRC cells.

The inhibition of BAMBI inhibits tumor growth and hepatic metastasis. To investigate the effects of BAMBI-siRNA on CRC growth and metastasis in vivo, xenograft mouse model was created by subcutaneous injection of HT-29 cells pretreated with BAMBI-siRNA or siRNA scramble or control to SPF nude mice. BAMBI-siRNA effectively suppressed tumor formation and tumor volume compared 
A
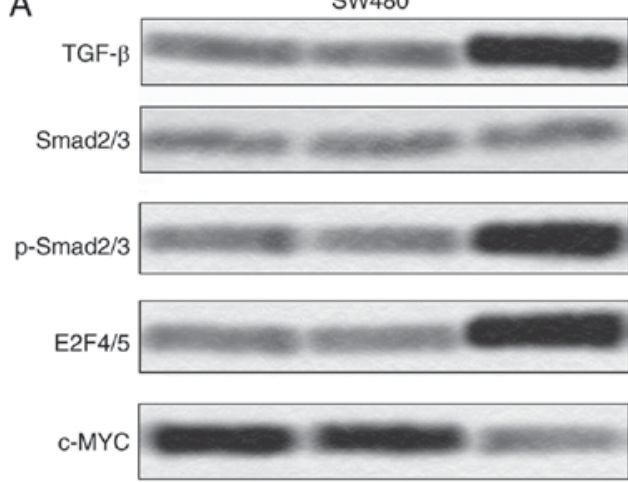

GAPDH

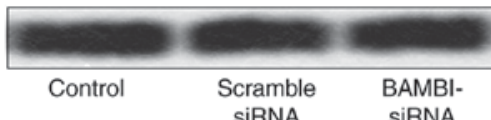

B

TGF- $\beta$

TGF- $\beta$
Smad $2 / 3$

HT-29

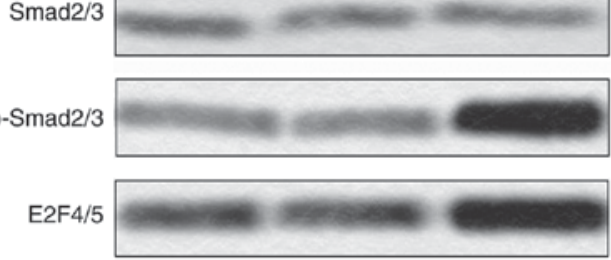

C-MYC
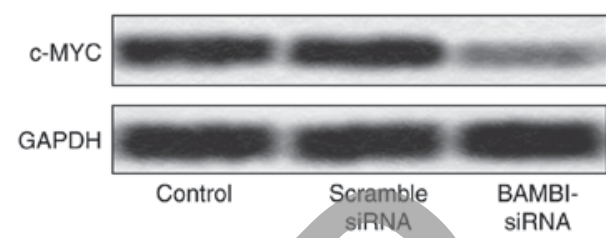

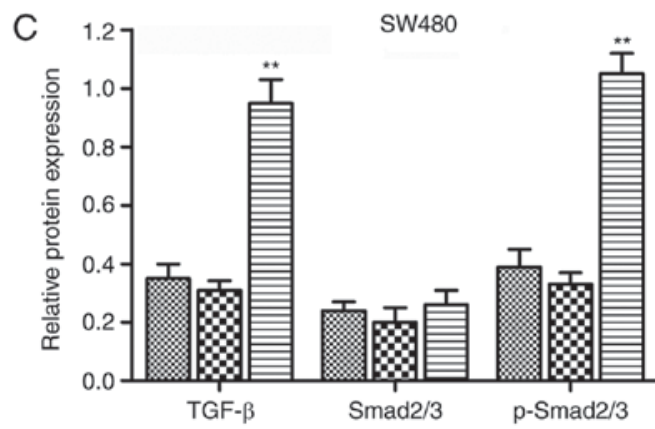

D
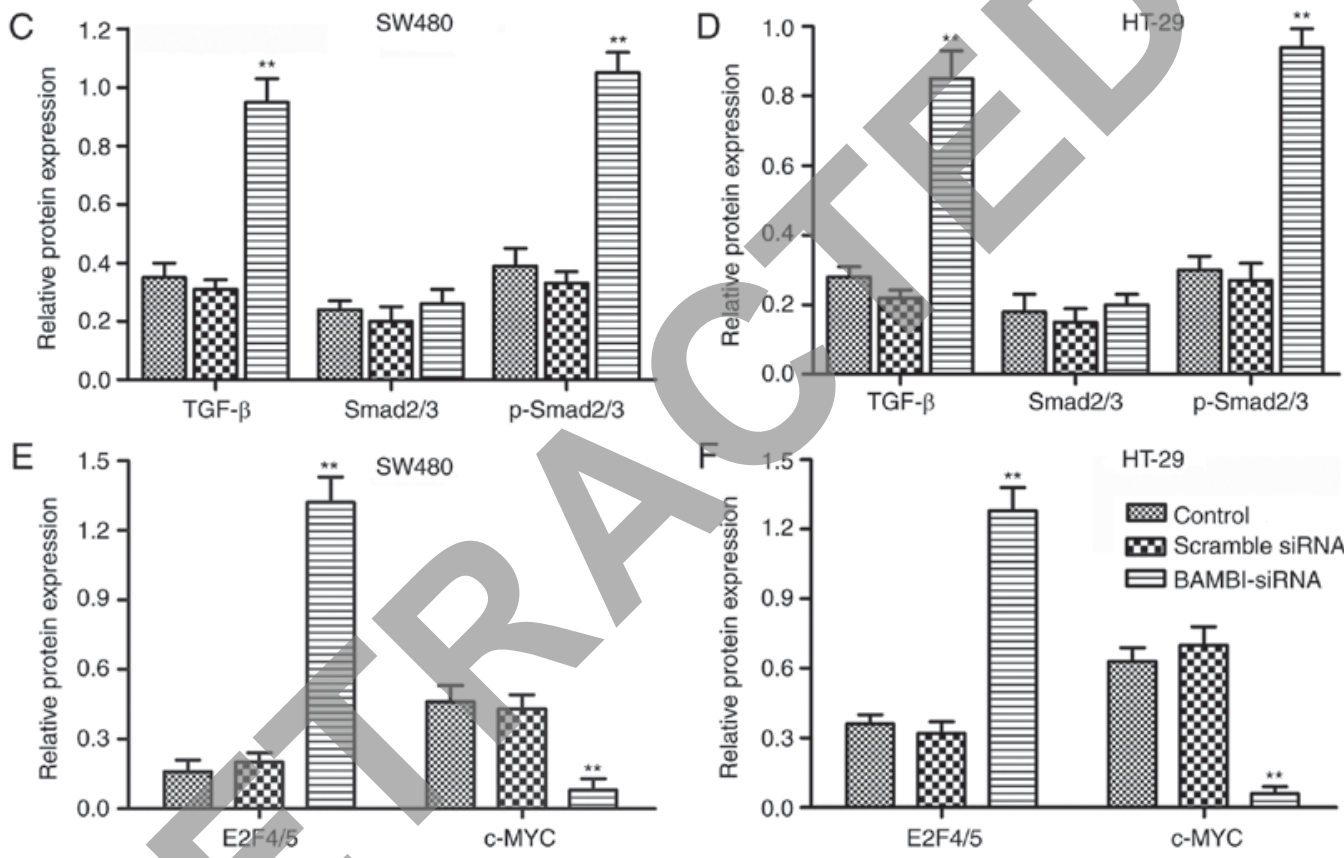

Figure 3. The inhibition of BAMBI activated TGF- $\beta /$ Smad signaling. CRC cell lines SW480 and HT-29 were transfected with BAMBI-siRNA or siRNA-scramble or control, respectively. (A and B) The expression of TGF- $\beta$ and substrate (Smad2/3 and p-Smad2/3) and downstream signaling molecules (E2F4/5 and c-MYC) in SW480 and HT-29 cells was detected through western blotting. GAPDH was used as an endogenous reference. (C and D) Histogram represents the statistical analysis of relative expression of TGF- $\beta$ and substrate (Smad2/3 and p-Smad2/3) in SW480 and HT-29 cells ("*P $<0.01$ vs. control group). (E and F) Histogram represents the statistical analysis of relative expression of E2F4/5 and c-MYC in SW480 and HT-29 cells (** P $<0.01$ vs. control group). The bars showed means \pm SD of three independent experiments.

with the control groups ( ${ }^{*} \mathrm{P}<0.05$, Fig. $4 \mathrm{~A}$ and B). The level of proliferation markers (Ki67 and PCNA) was also decreased in BAMBI-siRNA treated mice (Fig. 4C). These results suggested that BAMBI-siRNA restrained tumor growth of $\mathrm{CRC}$ in vivo. Besides that, fluorescent labeled HT-29 cells were found metastasized to liver. Fluorescence signal in liver was observed directly through IVIS Spectrum system (Fig. 4D). Moreover, the expression of BAMBI was suppressed by BAMBI-siRNA in vivo (Fig. 4E). Increased level of TGF- $\beta$, p-Smad2/3 and E2F4/5 and decreased expression of c-MYC was also detected in BAMBI-siRNA group mice (Fig. 4F). These changes in related proteins indicated that the inhibition of BAMBI activated TGF- $\beta /$ Smad signaling in vivo. These results indicated that the BAMBI-siRNA restrained the growth and hepatic metastases of CRC in vivo by activating the TGF- $\beta /$ Smad signaling pathway.

\section{Discussion}

Colon cancer is a highly metastatic cancer with more than 600,000 deaths each year (13). Clinical studies showed that most of the deaths were caused by metastatic CRC. In recent years, many efforts had been done on the development and metastasis of CRC, however, there is still a lot of research space for the potential regulation mechanisms up to now. In this study, we presented a new perspective that BAMBI regulated the viability and motility of CRC via the TGF- $\beta / \mathrm{Smad}$ pathway in vitro and in vivo.

Accumulated researches have suggested that BAMBI is involved in the pathogenesis of many human diseases. For example, BAMBI was verified to promote cell survival in hepatic stellate cells through Wnt/ $\beta$-catenin signaling (14). The elevated expression of BAMBI was identified to promote cell 

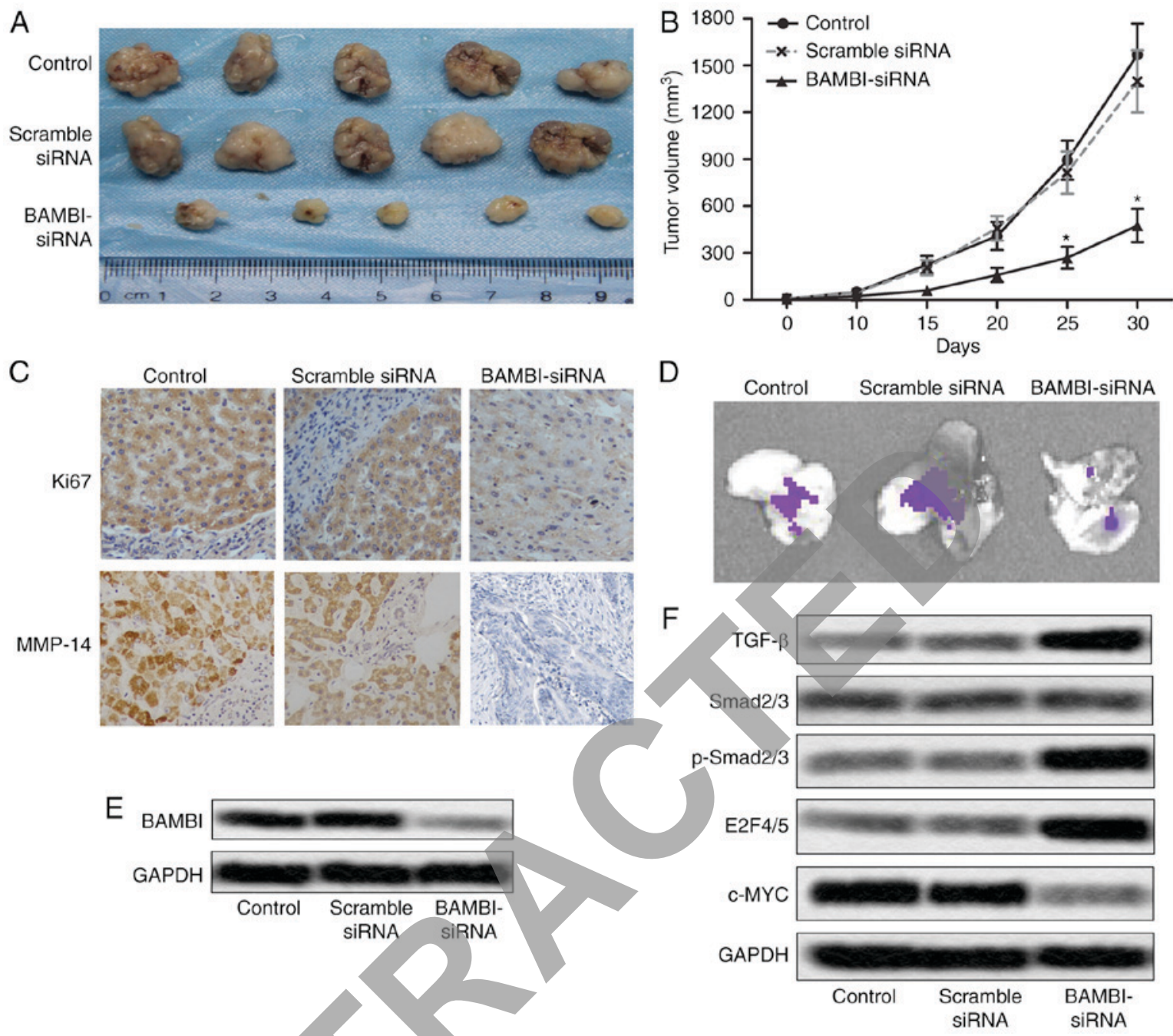

Figure 4. The inhibition of BAMBI inhibits tumor growth and hepatic metastasis. Fluorescently-labeled HT-29 cells were transfected with BAMBI-siRNA or siRNA-scramble or control. CRC xenograft mouse model was established by injecting the treated HT-29 cells to SPF nude mice subcutaneously. (A) Representative images of tumor in each group are presented $(n=5)$. (B) Tumor volume was calculated every 5 days after tumor formation and was shown as means $\pm \mathrm{SD}$ (" $\mathrm{P}<0.05$ vs. control group). (C) The expression of proliferation markers (Ki67 and PCNA) in formalin-fixed, paraffin-embedded tumors from BAMBI-siRNA group mice or siRNA-scramble or control group mice was detected through IHC analysis. (D) Livers from different groups were removed Fluorescence signal in liver was observed directly through IVIS Spectrum system. (E) The expression of BAMBI in BAMBI-siRNA/siRNA-scramble/control group mice was detected through western blotting. (F) The expression of TGF- $\beta$ and substrate (Smad2/3 and p-Smad2/3) and downstream signaling molecules (E2F4/5 and c-MYC) in BAMBI-siRNA/siRNA-scramble/control group mice was detected through western blotting. GAPDH was used as an endogenous reference. The bars showed means \pm SD of three independent experiments.

proliferation in non-small-cell lung cancer and hepatocellular carcinomas $(15,16)$. Additionally, high expression BAMBI and Smad7 may cooperatively inhibit the TGF- $\beta$ signaling, and thus promote the progression of gastric cancer (17). These researches indicate that reducing the level of BAMBI may help prevent the development of diseases. Thus, in our study, the expression of BAMBI was down-regulated by transfecting specific siRNA into CRC cell lines (SW480 and ST-29). Suppressed BAMBI effectively controlled the proliferation of SW480 and ST-29 cells and largely promoted cell apoptosis. Our study indicates that inhibition of BAMBI suppresses cell viability in CRC.

Numerous reports have indicated that BAMBI was participated in the metastasis of cancer. For example, BAMBI was evidenced to regulate the invasiveness and aggressiveness of bladder cancers through TGF- $\beta$ /BMP through autocrine and paracrine manner (18). Up-regulated expression of BAMBI was also found in gastric cancer and the knockdown of BAMBI suppressed metastasis of gastric cancer cells by inhibiting $\beta$-catenin and TGF- $\beta$ (19). In accordance with previous reports, BAMBI siRNA strongly reduced the would closing rate and the number of invasive cells compared with control group. Decreased expression of migration marker proteins further identify that BAMBI siRNA reduces cell motility of $\mathrm{CRC}$ in vitro.

The regulating role of TGF- $\beta /$ Smad signaling pathway has been identified in an enormous amount of researches. Antisense noncoding RNA in the INK4 locus (ANRIL, a long non-coding RNA located at chromosome 9p21) promoted the invasion and metastasis of thyroid cancer cells through TGF- $\beta /$ Smad signaling pathway (20). TGF- $\beta /$ Smad signaling pathway was identified as an important mechanism for NK cell immune evasion in childhood B-acute lymphoblastic leukemia (21). TGF- $\beta /$ Smad signaling is also involved 
in some other cancers, such as breast cancer (22), gastric carcinoma (23), including colon cancer (24). The interaction between BAMBI and TGF- $\beta /$ Smad pathway had been studied extensively, but the co-operation of the two in colorectal cancer has not been well studied. BAMBI was identified to inactivate TGF- $\beta$ signaling through the interaction with type I TGF- $\beta$ receptor (TGFBR1) receptors, thus preventing the formation of functional authentic receptor complexes (TGFRI/BMPRI and TGFRII/BMPRII) (25). In accordance with these previous reports, in our study, the expression of TGF- $\beta$ and activated $\mathrm{p}-\mathrm{Smad} 2 / 3$ was both up-regulated by BAMBI siRNA. Simultaneously, the level of nuclear effector molecules E2F4/5 and c-MYC was also changed in BAMBI siRNA group. These results above suggested that BAMBI activated the TGF- $\beta /$ Smad pathway and transmit signals through $\mathrm{Smad} 2 / 3$ in CRC.

In previous reports, BAMBI was reported to suppress tumor growth and metastasis in breast cancer by blocking the differentiation of human mesenchymal stem cells via inhibiting TGF- $\beta /$ Smad pathway (26). BAMBI was also identified to suppress tumor growth by down-regulating both $\beta$-catenin and TGF- $\beta 1$ signaling in hepatocellular carcinoma (27). Similar conclusion was verified in our study subsequently. Compared with the control mice, the BAMBI siRNA model mice exhibited restrained tumor growth and liver metastasis with enhanced expression of TGF- $\beta$ signaling pathway proteins. Results above indicate that BAMBI siRNA inhibited CRC growth and metastasis in vivo through activating TGF- $\beta /$ Smad pathway.

In conclusion, this study demonstrated that the inhibition of BAMBI restrained CRC growth and metastasis in vivo and in vitro. Besides, BAMBI exerted regulating role may through activating TGF- $\beta$ signaling pathway. Thus, our study suggests that BAMBI may be a potential target for future prevention and treatment of human $\mathrm{CRC}$

\section{Acknowledgements}

This work was funded by Natural Science Foundation of Hubei Province (grant no. 2013BKBO13).

\section{References}

1. Siegel RL, Miller KD and Jemal A: Cancer statistics, 2016. CA Cancer J Clin 66: 7-30, 2016.

2. Pugh SA, Shinkins B, Fuller A, Mellor J, Mant D and Primrose JN: Site and stage of colorectal cancer influence the likelihood and distribution of disease recurrence and postrecurrence survival: Data from the FACS randomized controlled trial. Ann Surg 263 1143-1147, 2016.

3. Parkin DM, Bray F, Ferlay J and Pisani P: Global cancer statistics, 2002. CA Cancer J Clin 55: 74-108, 2005.

4. Wolpin BM and Mayer RJ: Systemic treatment of colorectal cancer. Gastroenterology 134: 1296-1310, 2008.

5. Onichtchouk D, Chen YG, Dosch R, Gawantka V, Delius H, Massagué $\mathrm{J}$ and Niehrs C: Silencing of TGF-beta signalling by the pseudoreceptor BAMBI. Nature 401: 480-485, 1999.

6. Degen WG, Weterman MA, van Groningen JJ, Cornelissen IM, Lemmers JP, Agterbos MA, Geurts van Kessel A, Swart GW and Bloemers HP: Expression of nma, a novel gene, inversely correlates with the metastatic potential of human melanoma cell lines and xenografts. Int J Cancer 65: 460-465, 1996.

7. Sasaki T, Sasahira T, Shimura H, Ikeda S and Kuniyasu H: Effect of Nma on growth inhibition by TGF-betaa in human gastric carcinoma cell lines. Oncol Rep 11: 1219-1223, 2004.
8. Sekiya T, Adachi S, Kohu K, Yamada T, Higuchi O, Furukawa Y, Nakamura $\mathrm{Y}$, Nakamura $\mathrm{T}$, Tashiro $\mathrm{K}$, Kuhara S, et al: Identification of BMP and activin membrane-bound inhibitor (BAMBI), an inhibitor of transforming growth factor-beta signaling, as a target of the beta-catenin pathway in colorectal tumor cells. J Biol Chem 279: 6840-6846, 2004.

9. Oft $\mathrm{M}$, Heider $\mathrm{KH}$ and Beug $\mathrm{H}$ : TGFbeta signaling is necessary for carcinoma cell invasiveness and metastasis. Curr Biol 8: 1243-1252, 1998

10. Hata A, Shi Y and Massagué J: TGF-beta signaling and cancer: Structural and functional consequences of mutations in Smads. Mol Med Today 4: 257-262, 1998.

11. Grady WM, Myeroff LL, Swinler SE, Rajput A, Thiagalingam S, Lutterbaugh JD, Neumann A, Brattain MG, Chang J, Kim SJ, et al: Mutational inactivation of transforming growth factor beta receptor type II in microsatellite stable colon cancers. Cancer Res 59: 320-324, 1999.

12. Bierie B and Moses HL: Tumour microenvironment: TGFbeta: The molecular Jekyll and Hyde of cancer. Nat Rev Cancer 6: 506-520, 2006.

13. Ferlay J, Shin HR, Bray F, Forman D, Mathers C and Parkin DM: Estimates of worldwide burden of cancer in 2008: GLOBOCAN 2008. Int J Cancer 127: 2893-2917, 2010.

14. Subramaniam N, Sherman MH, Rao R, Wilson C, Coulter S, Atkins AR, Evans RM, Liddle C and Downes M: Metformin-mediated Bambi expression in hepatic stellate cells induces prosurvival Wnt $/ \beta$-catenin signaling. Cancer Prev Res (Phila) 5: 553-561, 2012.

15. Miao S, Zhao L, Gao J, Wang H and Cui Z: Distribution and mRNA Expression of BAMBI in Non-small-cell Lung Cancer. Zhongguo Fei Ai Za Zhi 12: 203-207, 2009 (In Chinese).

16. Lin Z, Gao C, Ning Y, He X, Wu W and Chen YG: The pseudoreceptor BMP and activin membrane-bound inhibitor positively modulates Wnt/beta-catenin signaling. J Biol Chem 283: 33053-33058, 2008.

17. Zhang Y, Yu Z, Xiao Q, Sun X, Zhu Z, Zhang J, Xu H, Wei M and Sun M: Expression of BAMBI and its combination with Smad7 correlates with tumor invasion and poor prognosis in gastric cancer. Tumour Biol 35: 7047-7056, 2014.

18. Khin SS, Kitazawa R, Win N, Aye TT, Mori K, Kondo T and Kitazawa S: BAMBI gene is epigenetically silenced in subset of high-grade bladder cancer. Int J Cancer 125: 328-338, 2009.

19. Liu K, Song X, Ma H, Liu L, Wen X, Yu J, Wang L and Hu S: Knockdown of BAMBI inhibits $\beta$-catenin and transforming growth factor beta to suppress metastasis of gastric cancer cells. Mol Med Rep 10: 874-880, 2014.

20. Zhao JJ, Hao S, Wang LL, Hu CY, Zhang S, Guo LJ, Zhang G, Gao B, Jiang Y, Tian WG and Luo DL: Long non-coding RNA ANRIL promotes the invasion and metastasis of thyroid cancer cells through TGF-beta/Smad signaling pathway. Oncotarget 7: 57903-57918, 2016.

21. Rouce RH, Shaim H, Sekine T, Weber G, Ballard B, Ku S, Barese C, Murali V, Wu MF and Liu H: The TGF- $3 /$ SMAD pathway is an important mechanism for NK cell immune evasion in childhood B-acute lymphoblastic leukemia. Leukemia 30: 800-811, 2016.

22. Jiang HL, Sun HF, Gao SP, Li LD, Hu X, Wu J and Jin W: Loss of RAB1B promotes triple-negative breast cancer metastasis by activating TGF- $\beta /$ SMAD signaling. Oncotarget 6: 16352-16365, 2015.

23. Yu D, Shin HS, Lee YS and Lee YC: miR-106b modulates cancer stem cell characteristics through TGF- $\beta / S$ Smad signaling in CD44-positive gastric cancer cells. Lab Invest 94: 1370-1381, 2014.

24. Zhang B, Halder SK, Kashikar ND, Cho YJ, Datta A, Gorden DL and Datta PK: Antimetastatic role of Smad4 signaling in colorectal cancer. Gastroenterology 138: 969-980.e1-e3, 2010.

25. Togo N, Ohwada S, Sakurai S, Toya H, Sakamoto I, Yamada T, Nakano T, Muroya K, Takeyoshi I, Nakajima T, et al: Prognostic significance of BMP and activin membrane-bound inhibitor in colorectal cancer. World J Gastroenterol 14: 4880-4888, 2008.

26. Shangguan L, Ti X, Krause U, Hai B, Zhao Y, Yang Z and Liu F: Inhibition of TGF- $\beta /$ Smad signaling by BAMBI blocks differentiation of human mesenchymal stem cells to carcinoma-associated fibroblasts and abolishes their protumor effects. Stem Cells 30: 2810-2819, 2012.

27. LeeS,LeeMJ,Zhang J,Yu GR and Kim DG: C-terminal-truncated HBV X promotes hepato-oncogenesis through inhibition of tumor-suppressive $\beta$-catenin/BAMBI signaling. Exp Mol Med 48: e275, 2016. 